\title{
ARQUEOMETRÍA DEL ARTE: ESTUDIOS FISICOQUÍMICOS DE PIGMENTOS ARQUEOLÓGICOS
}

\section{ARCHAEOMETRY OF ART: PHYSICAL AND CHEMICAL STUDIES OF ARCHAEOLOGICAL PIGMENTS}

\author{
Mariel A. LÓPez* Guillermo A. de la \\ FUENTE* * \& DÁNAE FIORE***
}

En este trabajo presentamos un panorama de los estudios de arqueometría de pigmentos arqueológicos desarrollados en la República Argentina a partir de la revisión y la cuantificación bibliométrica de trabajos presentados en los congresos y reuniones nacionales e internacionales sobre arqueometría, llevadas a cabo en el sur de Sudamérica. En segundo lugar, analizamos los trabajos que forman parte de este volumen, comentando las principales técnicas aplicadas, así como los resultados e interpretaciones a las cuales han llegado los colegas que han participado del IV Congreso Argentino de Arqueometría efectuado en 2011 en Luján, evaluando sus alcances y limitaciones y destacando los frutos del trabajo interdisciplinario.

Palabras clave: arqueometría, pigmentos, técnicas, resultados, contextos

In this paper we present an overview of archaeometric research of archaeological pigments conducted in Argentina through the review and bibliometric analysis of papers presented at national and international archaeometry conferences and meetings held in the Southern Cone of South America. Examining the issues addressed by the papers included in this volume, we comment on the techniques used and on the results and interpretations developed by colleagues participating in the IV Congreso Argentino de Arqueometria, beld in 2011 in Luján. We assess their contributions and limitations and emphasize the benefits of interdisciplinary research.

Key words: archaeometry, pigments, techniques, results, contexts

\section{INTRODUCCIÓN}

El objetivo de este trabajo es presentar una síntesis crítica sobre los avances realizados en los estudios arqueométricos de pigmentos arqueológicos en el sur de Sudamérica, a partir de los ejemplos presentados en diversos congresos y reuniones científicas y, en especial, a partir de los trabajos reunidos en este volumen.

En líneas generales existe un consenso en aceptar que el término arqueometría se impuso a partir de la aparición de la revista Archaeometry, fundada en 1958 por el Research Laboratory for Archaeology and the History of Art de la Universidad de Oxford. En su origen, el foco de la arqueometría estuvo restringido básicamente a los análisis de tipo cuantitativos y fisicoquímicos sobre materiales arqueológicos, aunque muchas de las instituciones de origen también estuvieron vinculadas al arte y a la conservación (Aitken 1961; Olin 1982, en Montero Ruiz et al. 2007). Posteriormente, y en especial a partir de la aparición de la revista Journal of Archaeological Science fundada en 1974, predominaron los análisis biológico-zoológicos y botánicos por sobre los análisis de caracterización de materiales, aunque con el tiempo estos últimos fueron adquiriendo una mayor relevancia.

* Mariel Alejandra López, Instituto de Arqueología, Facultad de Filosofía y Letras, Universidad de Buenos Aires, 25 de mayo $217,3^{\circ}$ piso, of. 3 (1002), Ciudad Autónoma de Buenos Aires, Argentina. Consejo Nacional de Investigaciones Científicas y Técnicas, email: marielarqueologia@yahoo.com.ar

* Guillermo Adrián de la Fuente, Laboratorio de Petrología y Conservación Cerámica, Escuela de Arqueología, Universidad Nacional de Catamarca, Belgrano 300, Campus Universitario (4700), Catamarca, Argentina. Consejo Nacional de Investigaciones Científicas y Técnicas, email: gfuente2004@ yahoo.com.ar

** Dánae Fiore, Asociación de Investigaciones Antropológicas, Bartolomé Mitre 1131 7"G" (1036), Ciudad Autónoma de Buenos Aires, Argentina Universidad de Buenos Aires. Consejo Nacional de Investigaciones Científicas y Técnicas, email: danae_fiore@yahoo.es 
Los estudios arqueométricos tienen sus antecedentes en análisis de composición de diversos tipos de materiales arqueológicos (metales, cerámica, roca, vidrio, entre los principales) que datan de fines del siglo XVIII y en estudios de arqueobotánica y arqueozoología que datan desde principios del siglo xIx (Aitken 1961; Tite 1972; Leute 1987; Pollard \& Heron 1996; Maggetti 2006; Montero Ruiz et al. 2007; Pollard et al. 2007). En líneas generales, y según Pernicka (1998), pueden distinguirse tres etapas en el desarrollo de los mismos: la Formativa (siglo xIx a 1930), la de Desarrollo (1930 a 1970) y la de Expansión (1970 en adelante). Durante la primera etapa se iniciaron, básicamente, los estudios radiográficos o de rayos $\mathrm{X}$ y la fotografía aérea para prospección y registro. La segunda etapa se caracterizó por ser la época en que se desarrollaron las principales técnicas de medición como, por ejemplo, la espectrometría de emisión óptica, el arqueomagnetismo, la datación por radiocarbono, la prospección geofísica, los análisis por activación neutrónica, la datación por termoluminiscencia, la espectroscopia de absorción atómica y la fluorescencia de energías de dispersión por rayos X, entre las principales. La tercera etapa, en tanto, se caracterizó por ser la época en que se dio el mayor avance de las técnicas multielementales, especialmente la datación radiocarbónica por AMS (espectrometría de masas por acelerador), la teledetección espacial, los sistemas de información geográfica y la minería de datos o data mining (Montero Ruiz et al. 2007).

El uso de estas técnicas propició, en cierto modo, no solo el avance de los estudios de caracterización, sino, además, la investigación de la procedencia de los materiales u objetos y el uso de múltiples variables de análisis en conjunto. A partir de estos datos, las preguntas arqueológicas a responder en esta última etapa se concentraron mayormente en temas vinculados a las fuentes de aprovisionamiento, los rangos de acción, el comercio y el intercambio entre grupos humanos (Tite 1991; Pollard \& Heron 1996; Pernicka 1998; entre otros).

En cuanto a los análisis de pigmentos arqueológicos en particular, hacia fines de la etapa Formativa y especialmente durante la etapa de Desarrollo se utilizó principalmente la técnica de difracción de rayos X para la identificación de los componentes cristalinos que actúan como cromóforos (colorantes) y/o aditivos de la fracción inorgánica de los pigmentos. Durante la etapa de Expansión fue posible caracterizar composicional y estructuralmente las mezclas pigmentarias con mayor precisión, tanto en su fracción inorgánica -sumando a la DRX técnicas tales como MEB-EDS y FT-IR-, como en su fracción orgánica -mediante técnicas tales como FT-IR, CG y CG-EM-, lo cual permitió identificar las sustancias utilizadas como ligantes en la mezcla pigmentaria (De la Fuente et al. 2012 Ms).

En la actualidad, es claro que resulta crucial integrar los resultados obtenidos en los análisis arqueométricos con los argumentos teóricos que explican los problemas arqueológicos. En otras palabras, un análisis solo aporta un dato, pero el dato necesariamente debe responder a una pregunta más allá de la descripción detallada de una muestra (sea de un artefacto, estructura o residuo) y esa pregunta es siempre de índole teórica, porque vincula conceptos, expectativas, indicadores y variables para resolver problemas específicos. En este sentido, hoy en día se tiende a adoptar un concepto más amplio para definir a la arqueometría, que exceda lo exclusivamente técnico-metodológico y abarque dichos aspectos teóricos.

Dado que en Argentina no existen hasta el momento laboratorios especializados en arqueometría que brinden servicios "a terceros", el desarrollo de estudios arqueométricos se ha gestado a partir de colaboraciones entre arqueólogos y especialistas de otras disciplinas; químicos, físicos, geólogos, entre los principales. Ello, sumado a que aún no existen protocolos de trabajo estandarizados y consensuados entre los distintos investigadores, ha generado que en algunos casos se generen problemas de comprensión y comunicación de los arqueólogos entre sí y entre arqueólogos y científicos de otras disciplinas. Dichos inconvenientes han sido parcialmente subsanados, precisamente, a partir del trabajo en colaboración interdisciplinaria, que ha generado la necesidad de aumentar la precisión de los requerimientos técnicos para la extracción y el procesamiento de muestras, así como para la interpretación de resultados. Asimismo, la presentación de las investigaciones realizadas en congresos también ha sido un factor estimulante de la comunicación intra e interdisciplinar, puesto que ha permitido poner de manifiesto los usos, los alcances y las limitaciones de cada técnica empleada. En tal sentido, este volumen intenta representar una contribución a este proceso interdisciplinario.

A partir del año 2005 en la Argentina se han desarrollado cuatro congresos nacionales de arqueometría (CAA). El primer CAA se realizó en la ciudad de Rosario en el año 2005. El segundo, que se llevó conjuntamente con el Primer Congreso Latinoamericano de Arqueometría (CLA), se realizó en Buenos Aires en el año 2007. El tercer CAA, que se llevó a cabo junto con las II Jornadas Nacionales de Bienes Culturales (JNBC), se realizó en Córdoba en el año 2009. En 2010, en el marco del XVII Congreso Nacional de Arqueología Argentina llevado a cabo en la ciudad de Mendoza, tuvimos la oportunidad de coordinar el simposio "Estudios arqueométricos de pigmentos: Contextos, artefactos y residuos", que se 


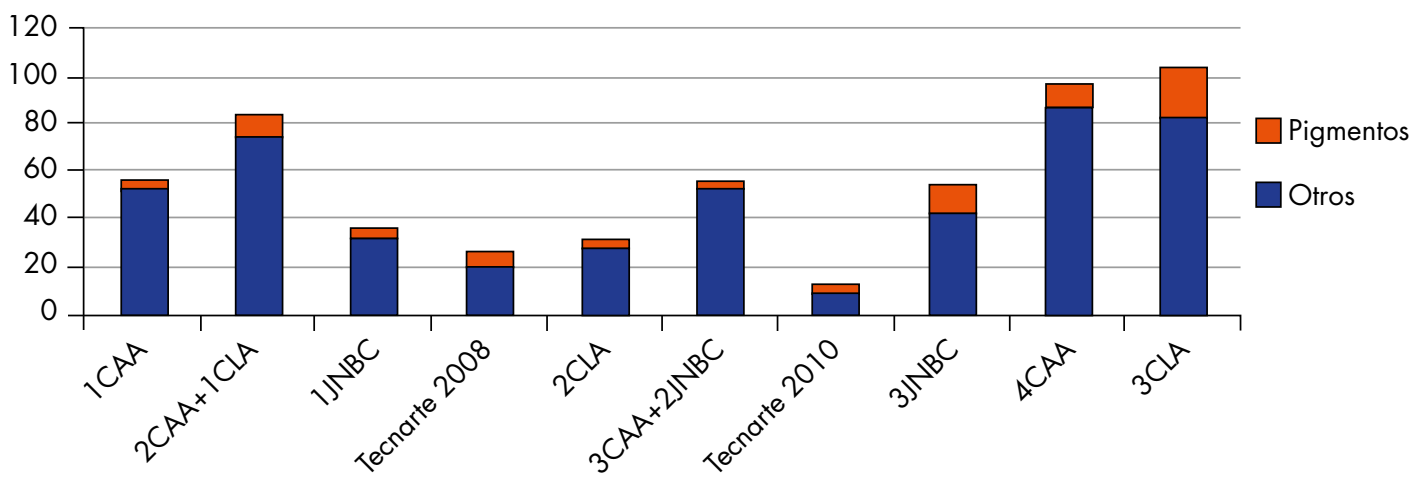

Gráfico 1. Trabajos de arqueometría de pigmentos en congresos argentinos y latinoamericanos.

Graph 1. Pigment archaeometry papers presented at Latin American and Argentinean conferences.

Tabla 1. Fuentes consultadas y cuantificadas para el cálculo de los índices y la preparación del gráfico. Table 1. Sources consulted and quantified to calculate the indexes and prepare the graph.

\begin{tabular}{|c|c|c|c|}
\hline Reunión & Referencia & Índices \\
\hline I CAA, Rosario, 2005 & Pifferetti \& Bolmaro 2005 & $3 / 56$ & 0,1 \\
\hline II CAA y I CLA, Buenos Aires, 2007 & Palacios et al. 2007 & $10 / 84$ & 0,1 \\
\hline I JNBC, Bariloche, 2007 & Vázquez \& Palacios 2007 & $3 / 36$ & 0,1 \\
\hline Tecnarte, Buenos Aires, 2008 & Libro de resúmenes & $5 / 26$ & 0,2 \\
\hline II CLA, Lima, 2009 & Vega Centeno et al. 2011 & $4 / 32$ & 0,1 \\
\hline III CAA y II JNBC, Córdoba, 2009 & Bertolino et al. 2010 & $3 / 56$ & 0,1 \\
\hline Tecnarte, Buenos Aires, 2010 & Libro de resúmenes & $5 / 13$ & 0,4 \\
\hline III JNBC, Buenos Aires, 2011 & Libro de resúmenes & $13 / 55$ & 0,2 \\
\hline IV CAA, Buenos Aires, 2011 & Libro de resúmenes & $9 / 95$ & 0,1 \\
\hline III CLA, Arica, 2011 & Gutiérrez et al. 2011 & $22 / 104$ & 0,2 \\
\hline
\end{tabular}

constituyó como el primer simposio de un congreso nacional de arqueología centrado exclusivamente en este tema. Posteriormente, en 2011 se llevó a cabo el cuarto CAA en la ciudad de Luján, durante el cual organizamos nuevamente un simposio centrado en la temática del análisis de pigmentos, titulado "Arqueometría de pigmentos: Casos arqueológicos y protocolos de investigación”; cinco de las ponencias allí presentadas dieron origen a los artículos publicados en este volumen.

Intercaladas con estos encuentros, también se llevaron a cabo las Jornadas Nacionales de Bienes Culturales, organizadas por investigadores de la Comisión Nacional de Energía Atómica (CNEA) en los años 2007, 2009 y 2011; así como las jornadas denominadas Tecnarte, organizadas por investigadores de la Facultad de Ingeniería de la Universidad de Buenos Aires y CNEA en los años 2008 y 2010. Sin ser de la magnitud de asistencia de los congresos nacionales, estos últimos encuentros constituyeron un importante impulso a la difusión de trabajos realizados en colaboración entre arqueólogos y analistas o científicos procedentes de las ciencias exactas y centrados en problemáticas arqueológicas o sobre bienes culturales en general.

Las mesas temáticas especialmente convocadas a lo largo de estos años en los congresos nacionales de arqueometría han rondado básicamente los siguientes temas: datación; prospección y otras aplicaciones en trabajos de campo; caracterización de materiales (biológicos, metálicos, cerámicos, pétreos, pigmentos, vidrios, etc.); preservación y conservación; estadística e informática aplicadas a la arqueología y arquitectura. En todos ellos se presentaron trabajos centrados en análisis de pigmentos.

El índice bibliométrico calculado sobre la base de la cantidad de trabajos relativos a pigmentos sobre el total de trabajos presentados/publicados en cada evento 
científico, arroja índices de entre 0,1 y 0,2 , con solo un caso de un índice de 0,4 trabajos de pigmentos sobre el total de trabajos (Tabla 1 y Gráfico 1).

Esto implica que la proporción de trabajos centrados en estudios de materiales pigmentarios no aumentó ni disminuyó con el tiempo, sino que, a lo largo de estos eventos científicos, se ha mantenido sumamente estable. Sin embargo, lo que sí se ha evidenciado es un cambio en la cantidad y la variedad de técnicas empleadas sobre un mismo conjunto de muestras, en los cuidados relativos a los requerimientos sobre la extracción y el procesamiento de las muestras, así como una creciente complejidad en los tipos de preguntas de investigación realizadas acerca de los materiales bajo estudio. Este proceso puede evidenciarse claramente en los trabajos que constituyen este volumen.

\section{MATERIALES Y TÉCNICAS EN LA ARQUEOMETRIA DE PIGMENTOS: UN PANORAMA A PARTIR DE LOS TRABAJOS DE ESTE VOLUMEN}

Franco y colaboradores proponen en su trabajo analizar la utilización de pigmentos en los sitios Río Bote 1 y Huyliche 1, ubicados en la cuenca superior del río Santa Cruz (Patagonia Argentina), y evaluar si existieron cambios en el uso de dichos materiales entre el 3740 AP (fecha del primer sitio) y el 420 AP (fecha del segundo). Ambos sitios son enterratorios humanos: en Río Bote 1 se hallaron tres individuos con aplicación de pigmentos sobre los cuerpos, pero no en los sedimentos adyacentes, mientras que en el chenque Huyliche 1 se hallaron restos de por lo menos seis individuos con pigmento aplicado sobre los cuerpos, los sedimentos y las rocas del chenque.

Los autores realizaron análisis de los sedimentos adyacentes a los cuerpos con lupa binocular (también denominada como microscopio binocular o estereomicroscopio), señalando el porcentaje de hidróxidos y óxidos de hierro presentes en las muestras. Asimismo, realizaron difracción de rayos $\mathrm{X}$ a las muestras halladas sobre restos humanos en Río Bote 1 (por cuestiones de conservación en Huyliche 1 fue imposible realizar estos estudios) y sobre muestras de sedimentos, tanto adyacentes como alejados a los restos óseos en cada sitio.

Los autores sostienen que en Río Bote 1 el 75\% de las muestras posee menos del 10\% de hidróxidos y óxidos de hierro, mientras que en Huyliche 1, el 60\% de las muestras tiene más de un $40 \%$ de compuestos oxidados de hierro y que sus concentraciones varían de individuo en individuo, siendo además en este último sitio mayores en los individuos que en los sedimentos adyacentes. Respecto de la composición de las muestras pigmentarias, estas son variadas, e incluyen casos constituidos por "ocre, yeso y feldespato de tipo albita", y casos de "material colorante" más "hidroxiapatita" (presumiblemente proveniente del hueso humano en el que se presenta el pigmento) y "calcita".

A partir de esta información, los autores infieren la existencia de un cambio en la forma de aplicación de pigmentos a lo largo del período estudiado, que pasan de ser utilizados directamente sobre los cuerpos, probablemente envueltos en material orgánico antes de ser enterrados en un sitio a cielo abierto (Río Bote 1), a colocarlos tanto sobre los cuerpos como sobre los sedimentos adyacentes y rocas que cubren al mismo, dentro de los límites del chenque (Huyliche 1).

A continuación, el trabajo de Tomasini y colaboradoras tiene por objetivo estudiar la preservación de imágenes rupestres pintadas en cueva de La Salamanca (Provincia de Catamarca, NOA), mediante análisis fisicoquímicos de dos muestras que permiten identificar sus procesos de alteración posteriores a la producción de los motivos. Las autoras detallan las condiciones observables de preservación del sitio, mencionan de manera muy somera la forma de extracción de las muestras y combinan una importante variedad de técnicas para su análisis: estereomicroscopio, microscopio con fuentes de luz visible y ultravioleta en modos normal y polarizado, microscopio electrónico de barrido de campo ambiental (FE-SEM) acoplado con un microanalizador por espectroscopia de dispersión de energía (SEM-EDS), espectrómetro con transformada de Fourier (FTIR) y espectroscopia Raman. Luego de presentar una breve síntesis sobre los factores naturales y antrópicos, macroscópicos y microscópicos, que causan alteraciones y pueden conducir al deterioro de las pinturas, se presenta la determinación de la composición del pigmento rojo para luego caracterizar sus formaciones cristalinas.

La muestra del panel $N^{\circ} 6$ se dispone en una estratigrafía de tres capas, siendo la intermedia de coloración rojiza y compuesta por "hierro [...] que podría formar parte de una especie cromófora como la hematita $\left(\mathrm{Fe}_{2} \mathrm{O}_{3}\right)$ ", mientras que las zonas blancas están compuestas por "calcio y azufre, lo cual es indicativo de yeso $\left(\mathrm{CaSO}_{4} \cdot 2 \mathrm{H}_{2} \mathrm{O}\right)$ ”, que -según análisis petrográficos- no es un compuesto proveniente del soporte rocoso.

La muestra del panel $\mathrm{N}^{\circ} 5$ es heterogénea y está compuesta por "calcio y azufre en una relación uno a uno característica de yeso". Asimismo se identifican "silicio, aluminio y metales alcalinos que sugieren la presencia de aluminosilicatos, posiblemente feldespatos de potasio (K) (ortoclasas), magnesio $(\mathrm{Mg})$ y sodio $(\mathrm{Na})$ 
(plagioclasas), en coincidencia con los resultados del análisis petrográfico de la roca soporte". A ello se suma un bajo "porcentaje atómico de hierro" que podría inferirse como indicativo de "presencia de hematita", y también "weddellita", una forma hidratada de oxalato de calcio.

A partir de estos resultados, las autoras infieren que en ambas muestras el compuesto responsable del color rojo es la hematita, mientras que la calcita es un componente minoritario, cuya concentración pudo haber disminuido con el tiempo debido a que "los carbonatos son sustancias básicas que pueden reaccionar con gases ácidos de la atmósfera”. Por lo tanto, debido a procesos de reacción ácido-base descritos detalladamente en el trabajo, el yeso podría haber reemplazado en forma gradual a la calcita y migrar desde la superficie exterior hacia la roca, envolviendo al pigmento por encima y por debajo. Dada dicha disposición estratigráfica del yeso y su ausencia en la composición mineralógica de la roca soporte, las autoras descartan que la presencia de este compuesto en las muestras sea el resultado de la incorporación intencional en la preparación de la mezcla pigmentaria o por infiltración del mineral a través de grietas. La procedencia del yeso es considerada entonces como resultante de la "acción de gases atmosféricos de origen natural volcánico que estuvieron presentes en el entorno de la cueva probablemente luego de haberse ejecutado las mismas", lo cual concuerda con su estado de descamación. Además, Tomasini y colaboradoras identifican a la weddellita como indicador de deterioro biológico de las pinturas, en tanto que su presencia sugiere que estas habrían tenido "contacto con líquenes cuyo crecimiento se favorece por la presencia de yeso".

Luego, el trabajo de Acevedo y colaboradoras presenta un análisis de los pigmentos de colores rojo y negro en la alfarería del Estilo Negro sobre Rojo, procedentes del sitio Pintoscayoc 1, localizado en la Quebrada de Humahuaca (Provincia de Jujuy, Argentina) a través de la aplicación de una combinación de técnicas analíticas como difracción de rayos X (DRX), espectroscopia Raman (RS) y microscopia electrónica de barrido con espectroscopia de energía dispersiva (SEM-EDS). El trabajo es un caso interesante de aplicación de diversas técnicas analíticas complementarias para la resolución de un problema arqueológico, como lo es el origen de los pigmentos que expresan los colores negros y rojos, y que fueron utilizados por los alfareros antiguos para decorar las vasijas cerámicas. Las autoras demuestran mediante DRX y RS que para el color rojo y rojo-morado se utilizó un precursor primario de origen inorgánico como la hematita $\left(\alpha-\mathrm{Fe}_{2} \mathrm{O}_{3}\right)$; aunque se observa también la presencia de estructuras de grafito microcristalino y magnetita $\left(\mathrm{Fe}_{3} \mathrm{O}_{4}\right)$. Con relación al color negro, y a mediante el examen por SEM-EDS, las autoras descartan la presencia de manganeso ( $\mathrm{Mn}$ ) como un probable precursor inorgánico y, con base en los resultados obtenidos básicamente por RS y DRX, ellas postulan la presencia de estructuras de grafito microcristalino, junto con la fase magnetita. Las bandas presentadas en las figuras $6 \mathrm{~b}$ (muestras 9 y 7 ) y $7 \mathrm{~b}$ (muestra 8) para el color negro son las comúnmente conocidas como band $-G\left(1585 \mathrm{~cm}^{-1}\right)$ y band-D $\left(1350 \mathrm{~cm}^{-1}\right)$, asignadas a estructuras de grafito desordenado, originadas en un precursor biogénico como los restos vegetales (plantas) (Van der Weerd et al. 2004; De la Fuente \& Pérez Martínez 2008). Esto hace pensar que el precursor del color negro puede haber sido un pigmento de origen biogénico u orgánico, mezclado con un coloide como vehículo constituido por aluminosilicatos (arcillas) para lograr su fijación precocción. Lo interesante de los resultados obtenidos para caracterizar el color negro es la presencia de una combinación de grafito y magnetita, lo cual plantea interrogantes mayores en torno a la tecnología de cocción. Dado que las autoras reportan una cocción oxidante para la alfarería es posible pensar no en un evento de bicocción stricto sensu, sino en un cambio intencional en las condiciones atmosféricas de cocción de condiciones oxidantes a reductoras, quizás en los últimos minutos del proceso, dado que está comprobado que la transformación de hematita (quizás contenida en el coloide que actúa como vehículo) en magnetita se produce a una temperatura de $375^{\circ} \mathrm{C}$ y con solo 15 minutos de exposición a la ausencia de oxígeno (Betancur et al. 2002). Además, también se observa la presencia de magnetita en el color rojo, posiblemente como un proceso incipiente de transformación en el cual coexisten las dos fases minerales (Betancur et al. 2002). El caso de estudio es muy interesante y requerirá de mayores análisis sobre este estilo cerámico para dilucidar si se trata de un procedimiento técnico intencional implementado por los alfareros en torno a la obtención del color negro.

El trabajo de Marte y colaboradoras, a continuación, presenta una aproximación innovadora para el estudio de los acabados de superficie y las características pigmentarias de los colores aplicados en los diseños de las superficies cerámicas del Estilo Negro y Blanco sobre Rojo, procedentes del sitio Pintoscayoc 1, característico de la Quebrada de Humahuaca (Provincia de Jujuy, Argentina) durante el Período de Desarrollos Regionales (ca. 1000-1480 DC). El estudio se basa en la aplicación de microscopia óptica (MO), espectroscopia Raman (RS) y análisis multiespectrales (AM); este último aplicado para estudiar y definir las características generales de la ejecución del proceso de pintado de las superficies 
cerámicas. Asimismo, como enfoque innovador se puede destacar el uso de la técnica estratigráfica para el estudio de las secuencias de ejecución de los diseños en secciones transversales de fragmentos de alfarería. Los análisis realizados permitieron comprobar, por un lado, la presencia de un engobe rojo-morado sobre el cual se aplicaron las pinturas de color negro y blanco y, por otro, mediante RS se pudo asignar el color rojo al dióxido de titanio $\left(\mathrm{TiO}_{2}\right)$ en su fase anatasa mezclado con otros compuestos de hierro (muestra $\mathrm{N}^{\circ} 12$ ) y la presencia de hematita para otra de las muestras analizadas (muestra $\mathrm{S} / \mathrm{N}$ ), así como asociar el color negro a la presencia de carbón (C). Finalmente, el color blanco no pudo determinarse analíticamente para la muestra $\mathrm{N}^{\circ} 12$, aunque los resultados de $\mathrm{RS}$ para la muestra $\mathrm{S} / \mathrm{N}$ indican la presencia del compuesto hidroxiapatita (hueso pulverizado). Con respecto al color blanco, los autores hipotetizan su aplicación poscocción sobre la base de las exfoliaciones observadas en algunos fragmentos, aunque son cautos y plantean que se requiere de análisis adicionales para corroborar esta hipótesis. Una observación interesante está relacionada con la presencia de una capa o estrato delgado translúcido, de espesor uniforme, que se observa únicamente por sobre la pintura de color blanco, lo cual hace pensar en la intencionalidad en la aplicación de esta capa a los efectos de proteger una pintura poscocción, o un efecto posdepositacional. Los estudios en estratigrafía de los cortes transversales de los fragmentos permitieron en general estudiar las características de los diferentes estratos o capas de pintura y diferenciarlas unas de otras y a su vez con respecto a la matriz. El resultado más concluyente de estos estudios es la determinación de un posible repinte en la muestra $\mathrm{N}^{\mathrm{o}} 13$ : una práctica escasamente estudiada para las cerámicas arqueológicas. El estudio de caso presentado por Marte y colaboradoras es una aproximación interdisciplinaria muy interesante al estudio de los engobes y pinturas en cerámicas arqueológicas, dado que el enfoque define las áreas pintadas como "superficies pictóricas" (sensu Maravelaki-Kalaitzaki \& Kallithrakas-Kontos 2003) y plantea una visión holística e integradora en el estudio de los diferentes colores intervinientes en la decoración de la cerámica arqueológica.

Finalmente, el trabajo de Bugliani y colaboradoras analizó "pigmentos" muestreados de los "diseños pintados" en distintos tonos de rojo y negro sobre materiales cerámicos del Período Formativo (300 AC-900 DC), Estilo Vaquerías. Se trata de un estilo de muy amplia dispersión en contextos domésticos aldeanos tempranos del norte argentino y chileno, pintado sobre el color de base de la pasta o, a veces, sobre un engobe crema/ blanco amarillento. El mismo representa la cerámica polícroma más antigua del Noroeste Argentino. Los materiales analizados en esta oportunidad proceden de los sitios Cardonal y Bordo Marcial, localizados en el Valle del Cajón, Departamento de Santa María de la Provincia de Catamarca, Argentina.

En esta ocasión las autoras aplicaron combinadamente espectroscopia Raman, difracción por rayos X (DRX) y microscopia electrónica de barrido con espectroscopia de energía dispersiva (SEM-EDX) a fin de identificar y caracterizar las materias primas con las que fueron pintados los diseños que se observan sobre seis fragmentos de este tipo de piezas cerámicas.

A partir de los resultados obtenidos, las autoras han determinado un predominio de hematita $\left(\mathrm{Fe}_{2} \mathrm{O}_{3}\right)$ en los pigmentos rojos y altos contenidos de manganeso ( $\mathrm{Mn}$ ), principalmente compuestos de jacobsita $\left(\mathrm{MnFe}_{2} \mathrm{O}_{4}\right)$ y bixbyta $\left(\mathrm{Mn}_{2} \mathrm{O}_{3}\right)$, junto con óxidos de hierro en la forma de magnetita $\left(\mathrm{Fe}_{3} \mathrm{O}_{4}\right)$ en los pigmentos negros. El análisis tecnológico no les permite confirmar a las autoras si el pigmento de color negro empleado fue aplicado de manera directa como mineral o si se emplearon minerales ricos en hierro y manganeso tratados térmicamente antes de su aplicación. No obstante ello, la complementariedad de las técnicas de análisis mencionadas, junto con los resultados de otras líneas de evidencia, principalmente derivadas de los estudios estilísticos y petrográficos, les permite sostener con bastante confianza que las piezas habrían sido pintadas antes de su cocción.

\section{COMENTARIOS FINALES}

Los trabajos reseñados y presentados en este volumen constituyen un claro ejemplo del avance efectuado en estos últimos años en las investigaciones sobre arqueometría de pigmentos arqueológicos en Argentina. Este avance es, en parte, producto y consecuencia de los distintos tipos de eventos organizados, puesto que estos no solo abren el espacio necesario para exponer resultados y discutir problemas prácticos enfrentados a lo largo del desarrollo de estas investigaciones, sino que, incluso, ponen el foco en el gran potencial que albergan estas pequeñas muestras arqueológicas. La mayoría de las veces estos eventos han sido organizados en conjunto con especialistas de otras disciplinas (particularmente químicos y físicos), y muestran los resultados del trabajo en colaboración, la complementariedad de técnicas aplicadas y la multiplicidad de líneas de evidencia que se ponen en juego a la hora de interpretar los datos analíticos. En este último sentido, la mayor compenetración de los especialistas de distintas disciplinas con el problema arqueológico constituye un factor crucial 
desde las primeras etapas de la investigación, puesto que la información obtenida suele resultar mucho más relevante y completa cuando el diseño del total del proyecto de investigación, desde las estrategias de muestreo pasando por las técnicas de laboratorio, hasta los análisis de los resultados, ha sido consensuado por todos los colegas involucrados en el estudio de cada caso. Es así que, cuando los físicos, químicos, ingenieros y/o geólogos involucrados en estas investigaciones comprenden los tipos de preguntas arqueológicas que pretendemos resolver, pueden muchas veces construir junto con los arqueólogos alternativas de mayor precisión y riqueza que cuando se les da exclusivamente el lugar para procesar muestras en el laboratorio sin participar en el resto del proceso de investigación.

Esperamos que este tipo de colaboración progrese en la constitución de equipos interdisciplinarios que favorezcan, a su vez, la formación de nuevos recursos humanos capaces de abordar los problemas de estudios arqueológicos.

\section{REFERENCIAS}

ArtKen, M. J., 1961. Physics and Archaeology. New York: Interscience. Bertolino, S.; R. CATTÁneo \& A. D. Izeta (Eds.), 2010. Libro de resúmenes del III Congreso Argentino de Arqueometría y II Jornadas Nacionales de Bienes Culturales, Córdoba, Argentina.

Betancur, J. D.; J. Restrepo, O. Arnache, J. Mazo-Zuluago, A. L. Morales, C. A. Barrero, J. J. Fernández, O. Pérez \& A. Bohórquez, 2002. Análisis Mossbauer de la transformación hematita a magnetita bajo tratamiento térmico en atmósfera controlada. Revista Colombiana de Física 34 (2): 410-414. Bogotá: Pontificia Universidad Javeriana.

De la Fuente, G. A. \& J. M. Pérez Martínez, 2008. Estudiando Pinturas en Cerámicas Arqueológicas "Aguada Portezuelo" ( $c a .600-$ 900 AD) del Noroeste Argentino: Nuevos Aportes a través de una aproximación arqueométrica por Microespectroscopia de Raman. Intersecciones en Antropologia 9: 173-186. Olavarría: Universidad Nacional del Centro de la Provincia de Buenos Aires.

De la Fuente, G. A.; D. Fiore \& M. A. López, 2012 Ms. Técnicas relevantes para la caracterización de pigmentos arqueológicos: sus alcances analíticos y sus aplicaciones en casos de Argentina.

Gutiérrez, S.; F. P. Hocquet, M. Rojas, M. Sepúlveda \& V. Silva Pinto (Eds.), 2011. Libro de resúmenes del III Congreso Latinoamericano de Arqueometría. Arica, Chile.
LeUTE, U., 1987. Archaeometry. An introduction to physical methods in archaeology and the history of art. Weinheim, N. Y.: VCH Publishers.

Libro de resúmenes del IV Congreso Argentino de Arqueometría, 2011. Luján: Universidad Nacional de Luján.

Libro de resúmenes de las III Jornadas Nacionales de Bienes Culturales, 2011. Buenos Aires: Comisión Nacional de Energía Atómica.

Libro de resúmenes Tecnarte 2008. Buenos Aires, Argentina.

Libro de resúmenes Tecnarte 2010. Buenos Aires, Argentina.

Maggettr, M., 2006. Archaeometry: quo vadis? Geological Society, Special Publications 257: 1-8. Londres: The Geological Society of London.

Maravelaki-Kalaitzaki, P. \& N. Kallithrakas-Kontos, 2003. Pigment and terracota analyses of Hellenistic figurines in Crete. Analytica Chimica Acta 497: 209-225. Amsterdan: Elsevier.

Montero Ruiz, I.; M. García Heras \& E. López Romero, 2007. Arqueometría: Cambios y tendencias actuales. Trabajos de Prebistoria 64 (1): 23-40. Madrid: CSIC.

Ous, J. S. (Ed.), 1982. Future directions in Archaeometry. A round table. Washington D. C.: Smithsonian Institution Press.

Palacios, O.; C. Vázquez, T. Palacios \& E. Cabanillas (Eds.), 2007. Libro de resúmenes del II Congreso Argentino de Arqueometría y I Congreso Latinoamericano de Arqueometría. Buenos Aires: Comisión Nacional de Energía Atómica.

PernicKa, E., 1998. Whiter metal analysis in archaeology? En L'atelier du bronzier en Europe du xx au vIII siècle avant notre ère. Actes du colloque international Bronze'96 Neuchâtel et Dijon, I: Les analyses de composition du métal: leur apport a l'archéologie de l'Âge du Bronze, C. Mordant, M. Pernot \& V. Rychnes, Eds., pp. 259-267. Paris: Éditions du Comité des Travaux Historiques et Scientifiques et du Centre de Recherches sur les techniques gréco-romaines de l'Université de Bourgogne.

PifferetTi, A. \& R. Bolmaro (Eds.), 2005. Libro de actas del I Congreso Argentino de Arqueometría. Rosario: Facultad de Humanidades y Artes, UNR.

Pollard, A. M. \& C. Heron, 1996. Archaeological Chemistry. Cambridge: Royal Society of Chemistry.

Pollard, M.; C. BatT, B. STern \& S. M. M. Young, 2007. Analytical Chemistry in Archaeology. Manuals in Archaeology. Cambridge: Cambridge University Press.

TrTe, M. S., 1972. Methods of Physical Examination in Archaeology. New York: Seminar Press.

- 1991. Archaeological Science. Past achievements and future prospects. Archaeometry 33 (2): 139-151. Oxford: University of Oxford.

Van der Weerd, J.; G. D. Smith, S. Firth; Y R. J. H. Clark, 2004. Identification of black pigments on prehistoric southwest american potsherds by infrared and Raman microscopy. Journal of Archaeological Sciences 31: 1429-1437.

Vázquez, C. \& O. Palacios (Eds.), 2007. Libro de actas de las I Jornadas Nacionales de Bienes Culturales. Bariloche, Argentina.

Vega Centeno, R.; P. Olivera \& S. Petrick, (Eds.), 2011. Libro de actas del II Congreso Latinoamericano de Arqueometría. Lima, Perú. 
